\title{
A new approach to solve perturbed symmetric eigenvalue problems
}

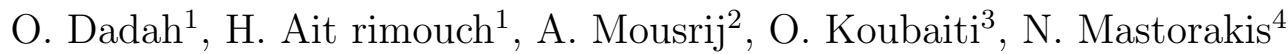 \\ ${ }^{1}$ Laboratory Physics and Material, University Moulay Slimane, Beni-Mellal \\ Morroco
}

${ }^{2}$ Material Physics Laboratory, Faculty of Sciences and Technology, Sultan Moulay Slimane University, Beni-Mellal
${ }^{3}$ Department of Mechanical engineering, FST, Sidi Mohammed Ben Abdellah University, Fez Morocco
${ }^{4}$ Hellenic Naval Academy, Pireus, Greece and Technical university of Sofia, Sofia
Bulgaria

Received: August 7, 2020. Revised: September 16, 2020. Accepted: September 25, 2020. Published: September $28,2020$.

\begin{abstract}
The objective of this study is to efficiently resolve a perturbed symmetric eigenvalue problem, without resolving a completely new eigenvalue problem. When the size of an initial eigenvalue problem is large, its multiple times solving for each set of perturbations can be computationally expensive and undesired. This type of problems is frequently encountered in the dynamic analysis of mechanical structures. This study deals with a perturbed symmetric eigenvalue problem. It propose to develop a technique that transforms the perturbed symmetric eigenvalue problem, of a large size, to a symmetric polynomial eigenvalue problem of a much reduced size. To accomplish this, we only need the introduced perturbations, the symmetric positive-definite matrices representing the unperturbed system and its first eigensolutions. The originality lies in the structure of the obtained formulation, where the contribution of the unknown eignsolutions of the unperturbed system is included. The effectiveness of the proposed method is illustrated with numerical tests. High quality results, compared to other existing methods that use exact reanalysis, can be obtained in a reduced calculation time, even if the introduced perturbations are very significant.
\end{abstract}

Keywords- Finite element method, Modal reanalysis, Perturbation, Polynomial eigenvalue problem, Structural modification, Truncated modal basis.

\section{INTRODUCTION}

$\mathbf{T}$ HE design of a mechanical structure process goes through the controlling and development of its dynamic characteristics. During the design phase, one conveys numerous iterations before ensuing the prototype. A substantial period of time is dedicated to contrasting the respective dynamic behaviours of the various alternatives of design. At each iteration, one compares the calculated eigensolutions of the real model with those of the desired one. For each structural modification, the same structure is treated with the form and the characteristic matrices which are simply updated.

To avoid long and arduous repetitions, efficient techniques of matrix perturbation labeled as the technique of reanalysis of dynamic structural modifications have been developed from a truncated modal base. The incomplete modal scheme, identified from the original structure, heavily influence the modified structure results.

Elliott and Mitchell [1], Coupry [2] and Ram and Braun 3] highlight the effects of modal truncation. In order to reduce these effects, several authors [4, 5, 6] proposed strategies allowing to enrich the identifed modal sub-basis. Other authors [6, 7, 8, 9] presented the effect of the static residues in a greater or lesser approximate form.

While I. G. Burova proposed in [10, 11] applications of local polynomial integro differential splines to solve the problem of calculating the real eigenvalues, based on either splines of Lagrangian type of fifth order, or polynomial integro-differential splines. I. G. Burova presented in [10] one of those applications in order to resolve fredholm equation. 12 proposed a reanalysis method of vibrations, based on a combined block approximation with shifting, using triangular factorizations in order to calculate the prorblem's eigenvalues. 13 focused one topological modificatiins by adding DoFs.

In this paper, we suggest a reanalysis method, which reduces the computation time through using data obtained either from an experiment or from a theoretical analysis based on a finite element model of the structure [14, 15, 16, 17. The originality lies in the fact that the strategy takes into account, in a very significant manner, the contribution of the unknown modes. This contribution will be represented by residual flexibility terms, which can be determined either through experiment or theoretically.

Nelson [18] presented a simplified procedure for calculating the derivatives of the eigenvectors of an arbitrary order system using only the eigenvectors of left and right 
and their associated eigenvalues. However, these expressions have been derived based only on the standard eigenvalue problem. Chen and Wada [19] formulated a first order perturbation solution using an expansion of the power series to perform a dynamic structural analysis. The first order of the proposed method has already been developed in [6] and compared to the technique developed in [7. In order to develop our method, various hypotheses have been made. The structure is presumed to be linear, undamped and fixed. The strategy can be extended to damped and floating structures.

\section{THEORY}

The theory of eigenvalues perturbations or the structural modification in the design of mechanical systems has been well studied. However, most articles have only applied this method to systems with small system disturbances as demonstrated by Fox and Kapoor 20. Our method consists of developing a formula to address disturbed systems during important modifications. Let us consider a system whose modes of vibration are governed by the solutions of the following generalized problem of the eigenvalue:

$$
\left[B_{0}\right] y_{0}=\lambda_{0}\left[A_{0}\right] y_{0}
$$

Where $\left[B_{0}\right]$ and $\left[A_{0}\right]$ denote the nominal matrices of the system, both of size $[n \times n], \lambda_{0}$ represents a nominal eigenvalue, and $y_{0}$ is the corresponding right nominal eigenvector. Let us Suppose that all the undisturbed eigenvalues of the system are distinct, and that $\left[B_{0}\right]$ and $\left[A_{0}\right]$ are symmetrical and positively defined. The left eigenvectors of the initial system, designated by $q_{0}^{T}$, satisfy:

$$
q_{0}^{T}\left[B_{0}\right] q_{0}=\lambda_{0} q_{0}^{T}\left[A_{0}\right] q_{0}
$$

We suppose that the left eigenvector $j^{\text {th }}$ and the right eigenvector $i^{\text {th }}$ have been correctly normalized so that they satisfy the following orthogonality conditions:

$$
q_{0 i}^{T}\left[A_{0}\right] q_{0 j}=\delta_{i j}, q_{0 i}^{T}\left[B_{0}\right] q_{0 j}=\lambda_{i j} \delta_{i j}
$$

Where $\delta_{i j}$ denotes the Kronecker delta and $i, j=$ $1, \ldots, n$. After an initial analysis, structural design modifications are introduced so that the system matrices become:

$$
[A]=\left[A_{0}\right]+[\delta A],[B]=\left[B_{0}\right]+[\delta B]
$$

Where $\left[A_{0}\right]$ and $\left[B_{0}\right]$ denote the undisturbed matrices, $[\delta A]$ and $[\delta B]$ are the disturbed matrices, and $[A]$ and $[B]$ are the highly disturbed matrices. In addition, the elements of $[\delta A]$ and $[\delta B]$ are supposed to be of an order of magnitude lower than those of $\left[A_{0}\right]$ and $\left[B_{0}\right]$.
Its eigenvalue problem, in physical coordinates, is given by:

$$
\left[B+\hat{\lambda}_{\nu} A\right] \hat{q}_{\nu}=0
$$

Where $\hat{q}_{\nu}$ and $\hat{\lambda}_{\nu}$ are respectively the $\nu^{\text {th }}$ eigenvector and the eigenvalue of the perturbed structure, and $\nu=1, \ldots, n$

To have the problem of the eigenvalue (5) in modal coordinates, we considers the following transformation of coordinates:

$$
\hat{q}_{\nu}=Q p_{\nu}
$$

Where $p_{\nu}$ is the generalized coordinate vector. We replace Equation (6) in Equation (5) we obtain the following Equation:

$$
\left[\Lambda+Q^{T} \delta B Q-\hat{\lambda}_{\nu}\left(I+Q^{T} \delta A Q\right)\right] p_{\nu}=0
$$

Where $\Lambda=\operatorname{diag} \lambda_{\nu}$ and the orthogonality properties (3) of the mode forms are used to eliminate the matrices from the original structure.

The solutions of the previous equation are exact if all the modes of the original structure are used. However, this condition is rarely verified and the solutions can only be approximate. Consequently, the first advantage of the modal representation is that the dynamics of the original structure can be adequately represented by few of its fundamental modes (lowest frequencies). In addition, it can be applied in a wide variety of experimental and analytical cases.

Our objective is therefore to evaluate the eigensolutions of the modified structure without resorting to a new exact and thus costly analysis. In other words, we would like to look for the best faster approximate solutions of equation (7), using only the identified modal parameters of the original structure.

To distinguish between the identified (subscript 1) and unknown (subscript 2) portions of the original structure eigenbasis, we partition the full matrices $Q, \Lambda$ and $p_{\nu}$ as:

$$
Q=\left[\begin{array}{ll}
Q_{1} & Q_{2}
\end{array}\right], \quad \Lambda=\left[\begin{array}{cc}
\Lambda_{1} & 0 \\
0 & \Lambda_{2}
\end{array}\right] \text { and } p_{\nu}=\left[\begin{array}{l}
p_{1 \nu} \\
p_{2 \nu}
\end{array}\right]
$$

where $Q_{1}$ and $\Lambda_{1}=\operatorname{diag}\left\{\lambda_{\nu} ; \nu=1,2, \ldots \ldots, m\right\}$ are assumed to be known, $Q_{2}$ and $\Lambda_{2}=$ $\operatorname{diag}\left\{\lambda_{\nu} ; \nu=m+1, \ldots, n\right\} \quad$ are unknown $p_{1 \nu}$ and $p_{2 \nu} m$ is the number of known modes $(m<n)$. With this decomposition, Eq. (8) becomes:

$$
\hat{q}_{\nu}=Q_{1} p_{1 \nu}+Q_{2} p_{2 \nu}
$$

Before proposing the method, we will briefly review the classical Rayleigh-Ritz approach using only the truncated modal basis of the original structure. 
Where, the $m$ first eigensolutions of the original structure satisfy the bi-orthogonal relations

$$
Q_{1}^{T} B Q_{1}=\Lambda_{1}, Q_{1}^{T} A Q_{1}=I_{m}
$$

A classical Ritz procedure expresses the $\nu^{\text {th }}$ eigenvector $\hat{q}_{\nu}$ of the modified structure on the known modal sub-basis $Q_{1}$ of the original structure, as

$$
\hat{q}_{\nu} \cong Q_{1} p_{1 \nu}
$$

In this case, approximate solutions are given by the following reduced linear eigenvalue problem of order $m$

$$
\left[\Lambda_{1}+Q_{1}^{T} \delta B Q_{1}-\hat{\lambda}_{\nu}\left(I_{m}+Q_{1}^{T} \delta A Q_{1}\right)\right] p_{1 \nu}=0
$$

In practice, the quality of the reanalysis provided by this strategy is often disappointing, since its imprecision, even for moderate structural modifications, exacerbates an already delicate procedure. We remark that the convergence toward the exact solution is monotonic, but generally slow for an increasing $m$. The perennial question is how many modes should be included in $\mathrm{Y} 1$ for having an adequate reanalysis.Coupry 22 demonstrated the sensitivity of the approximated solutions to truncation errors, while Ram and Braun [3] gave upper and lower bounds on the eigensolutions calculated with this procedure.

\section{TRANSFORMATION OF THE EIGENVALUE PROBLEM}

The suggested method transforms, in a well structured form, the $N^{\text {th }}$ order linear eigenvalue problem (4) to a $m^{t h}(m<N)$ order symmetric polynomial eigenvalue one.

After decomposing equation 10 we left two parts; a known part 1 and an unknown part 2 . The development of the equation leads us to the following form:

$$
\begin{array}{r}
{\left[\Lambda_{1}+Q_{1}^{T} \delta B Q_{1}-\hat{\lambda}_{\nu}\left(I_{m}+Q_{1}^{T} \delta A Q_{1}\right)\right] p_{1 \nu}} \\
+\left[Q_{1}^{T} \delta B Q_{2}-\hat{\lambda}_{\nu} Q_{1}^{T} \delta A Q_{2}\right] p_{2 \nu}=0 \\
{\left[Q_{2}^{T} \delta B Q_{1}-\hat{\lambda}_{\nu} Q_{2}^{T} \delta A Q_{1}\right] p_{1 \nu}+\left[\Lambda_{2}+Q_{2}^{T} \delta B Q_{2}\right.} \\
\left.-\hat{\lambda}_{\nu}\left(I_{N-m}+Q_{2}^{T} \delta A Q_{2}\right)\right] p_{2 \nu}=0
\end{array}
$$

The development of the equation leads us to the following form:

$$
\left[\Lambda_{1}+Q_{1}^{T} \delta B_{\lambda} Q_{1}-\hat{\lambda}_{\nu}\left(I_{1}+Q_{1}^{T} \delta A_{\lambda} Q_{1}\right)\right] p_{1 \nu}=0
$$

$$
Q_{2} p_{2 \nu}=-\alpha R_{1}\left(\delta B-\hat{\lambda}_{\nu} \delta A\right) Q_{1} p_{1 \nu}
$$

$$
\alpha=\left[I+R_{1} \delta B-\hat{\lambda}_{\nu} R_{1} A\right]^{-1}
$$

Where $\delta B_{\lambda}=\Delta B \alpha, \quad \delta A_{\lambda}=\Delta A \alpha+(\delta B-$ $\left.\hat{\lambda}_{\nu} \delta A_{0}\right) \alpha R_{1} M$ and $R_{1}=Q_{2} \Lambda_{2}^{-1} Q_{2}^{T}$

The disadvantages of the established equations are that the problem of eigenvalues of reduced order in Equation (8) is nonlinear and that the matrices $\delta B_{\lambda}$ and $\delta A_{\lambda}$ can be complicated and costly to evaluate, because they are functions of the inverse matrix $\alpha$. It contains explicitly the matrix $A_{0}$ of the original structure and will be calculated for each eigenvalue in the range of frequencies of interest and for each structural modification.

\section{TRANSFormation OF THE NON-LINEAR EIGENVALUE PROBLEM 12}

The method used to solve the general problem (12) requires that, in a frequency band of interest $\left[0, f_{\max }\right]$ where $f_{\max }$ must be less than or equal to the greatest natural frequency identified of the original structure, the matrix $\alpha$ must be approximated by an $n \times n$ polynomial matrix of $n$ degree:

$$
\alpha \cong \beta\left[I+\hat{\lambda}_{\nu} R_{1} A+\sum_{i=1}^{n} \hat{\lambda}_{\nu}^{i}\left(R_{1} A \beta\right)^{i}\right]
$$

Where $\beta=\left[I+R_{1} \delta B\right]^{-1}$

Thus, the problem of the nonlinear eigenvalue of Equation 12 can be rewritten as the following polynomial eigenvalue of $n$ degree:

$$
\begin{array}{r}
{\left[\Lambda_{1}+Q_{1}^{T} \widehat{\delta B} Q_{1}-\hat{\lambda}_{\nu}\left(I_{1}+Q_{1}^{T} L_{1} Q_{1}\right)\right.} \\
\left.-\sum_{i=2}^{n} \hat{\lambda}_{\nu}^{i}\left(Q_{1}^{T} L_{i} Q_{1}\right)\right] p_{1 \nu}=0
\end{array}
$$

where the matrices $L_{i}$, satisfy the recurrence relation

$$
L_{i}=L_{(i-1)} \cdot D_{1}+\ldots+L_{1} \cdot D_{(i-1)}-\widehat{\delta B} \cdot D_{i}
$$

where:

$$
\begin{array}{r}
L_{1}=\widehat{\delta B}-\widehat{\delta A} \cdot D_{1}, \widehat{\delta B}=\delta B \beta, \widehat{\delta A}=\delta A \beta \\
D_{i}=R_{i} \widehat{\delta A}-R_{(i+1)} \widehat{\delta B} \text { and } R_{i}=Q_{2} \Lambda_{2}^{-i} Q_{2}^{T}
\end{array}
$$

As mentionned in references [21, 22, 23, 24], standard computational algorithms are used for solving polynomial eigenvalue problems. As example: in MATLAB, polyeig function solves this type of problem.

With this approach and with a given level of required accuracy, the modal reanalysis is described by m modes and the order of the eigenvalue problem is reduced to $\mathrm{m}$ too.

Eq (19) helps calculate eigenvalues while eigenvectors of the modified structure are expressed, according to, by the following equation

$$
\hat{q}_{\nu}=\beta\left[I+\sum_{i=2}^{n} \hat{\lambda}_{\nu}^{i} C_{i}\right] Q_{1} p_{1 \nu}
$$




$$
C_{1}=A_{1}
$$

for $\mathrm{i}=2,3, \ldots, \mathrm{n}$

$$
C_{i}=D_{1} C_{(i-1)}+\ldots+D_{(i-1)} C_{1}+D_{i}
$$

The quality of the reanalysis is satisfying, even if the degree of the polynomial eigenvalue problem 19 is equal to 1 . Thus, in most cases, we have to solve a problem of reduced symmetric linear eigenvalue of $m^{t h}$ order. The proposed method takes into account, in a very significant manner, the contribution of the unknown modes. This contribution can be estimated, after a modal identification for an experimental model, and must be calculated for an analytical model.

\section{Calculation cost Reduction}

The matrices $\widehat{\delta B}$ and $L_{k}$, for $k=1,2, \ldots, n$, are symmetrical. Therefore, Equation (19) represents a nonlinear and symmetric eigenvalue problem. The main advantage of the new system is that it uses fewer arithmetic operations, taking full advantage of the symmetry.

In practice, the modifications affect only a reduced number, $\varepsilon(\varepsilon \ll n)$, of DOFs of the structure. However, $\delta B, \delta A$ and $L_{k}$ are sparse matrices. Most of the calculations involved in Equation $(19)$ will be performed with reduced square matrices $\varepsilon \times \varepsilon$. Thus, significant simplifications and a consequent reduction of the computation time can be obtained. To illustrate the reduction regime, it is convenient to distribute $\delta B, \delta A, R_{k}$ and $Q_{1}$ as follows:

$$
\begin{gathered}
\delta B=\left[\begin{array}{cc}
\delta B_{\varepsilon} & 0 \\
0 & 0
\end{array}\right], \delta A=\left[\begin{array}{cc}
\delta A_{\varepsilon} & 0 \\
0 & 0
\end{array}\right] \\
R_{k}=\left[\begin{array}{ll}
R_{k}^{\varepsilon \varepsilon} & R_{k}^{\varepsilon i} \\
R_{k}^{i \varepsilon} & R_{k}^{i i}
\end{array}\right] \text { and } Q_{1}=\left[\begin{array}{l}
Q_{1}^{\varepsilon} \\
Q_{1}^{i}
\end{array}\right]
\end{gathered}
$$

Where $\varepsilon$ and $i$ denote sets of modified and unmodified DOFs, respectively, $\delta B_{\varepsilon}$ and $\delta B_{\varepsilon}$ are the condensed modification matrices of the modified DOFs, $Q_{1}^{\varepsilon}$ is a subset of $Q_{1}$ with modified DOFs and $Q_{1}^{i}$ is the other subset of $Q_{1}$.

After several calculations [25, 26, 27], the matrix $\beta$ takes the following form:

$$
\begin{array}{r}
\beta=\left[\begin{array}{cc}
\hat{\beta} & 0 \\
-R_{1}^{i \varepsilon} \widehat{\delta B}_{\varepsilon} & I_{n-m}
\end{array}\right] \\
\hat{\beta}=\left[I_{\varepsilon}+R_{1}^{\varepsilon \varepsilon} \widehat{\delta B}_{\varepsilon}\right]^{-1} \text { and } \widehat{\delta B}_{\varepsilon}=\delta B_{\varepsilon} \hat{\beta}
\end{array}
$$

Equation 26 allows us to obtain the matrix $\beta$ by inverting only a matrix $\varepsilon \times \varepsilon$ instead of an $n \times n$ matrix, with the significant advantage from a computational point of view.
The introduction of equations (25) and (26) in equation 19 leads to the problem of the condensed polynomial eigenvalue:

$$
\begin{array}{r}
{\left[\Lambda_{1}+Q_{1}^{\varepsilon T} \widehat{\delta B}_{\varepsilon} Q_{1}^{\varepsilon}-\hat{\lambda}_{\nu}\left(I_{1}+Q_{1}^{\varepsilon T} \hat{L}_{1} Q_{1}^{\varepsilon}\right)\right.} \\
\left.-\sum_{k=2}^{n} \hat{\lambda}_{\nu}^{k}\left(Q_{1}^{\varepsilon T} \hat{L}_{k} Q_{1}^{\varepsilon}\right)\right] p_{1 \nu}=0
\end{array}
$$

\section{Numerical tests}

Two numerical simulation tests were carried out, to illustrate the approach developed above. The first test concerns the blocking of the DOFs of the structures free extremity, the second test consists of affecting the structure by parametric modifications.

To test the proposed method, finite element models are used to generate both the problem data and the reference eigensolutions of the modified structure.

The following criteria are used to assess errors in approximate eigensolutions:

$$
\begin{array}{r}
\delta f_{\nu}(i n \%)=\frac{\left|f_{\nu}^{e x}-\hat{f}_{\nu}\right|}{f_{\nu}^{e x}}, M A C_{\nu}=\left[\frac{\left|Y_{\nu}^{T e x} \hat{Y}_{\nu}\right|}{\left\|Y_{\nu}^{e x}\right\|\left\|Y_{\nu}^{e x}\right\|}\right]^{2} \\
\text { and } \Delta_{M A C_{\nu}}=\left(1-M A C_{\nu}\right) \times 100
\end{array}
$$

Where $f_{\nu}^{e x}$ means reference values, $\hat{f}_{\nu}$ means approximate values, $\widehat{f_{\nu}^{r e}}$ means approximate values of a smaller problem, and is the relative error, in \%, on the approximate natural frequency compared to that of the reference and is the value of the modal assurance criterion between the eigenvectors referenced and approximated of the mode. The ideal result will give a value $\Delta_{M A C_{\nu}}$ of about $0 \%$.

For the two examples, the reanalysis will be done by the first five natural frequencies $(m=5)$ of the original structure. We will note the natural frequency of these latter, the beam is modeled using 42 beam elements, which gives a total of 126 DOFs (with 3 DOFs per node).

Its characteristics are as follows: Young's modulus $E=0.499=109 \mathrm{Nm}^{-2}$, the density $=7800 \mathrm{kgm}^{-3}$, the moment of inertia $I=0.279=10-4 m^{4}$, the cross section $A=0.001 \mathrm{~m}^{2}$ and the length $L=1.5 \mathrm{~m}$. 
(a)

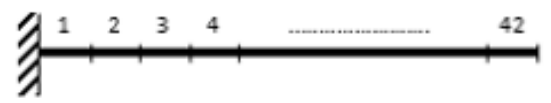

(b)

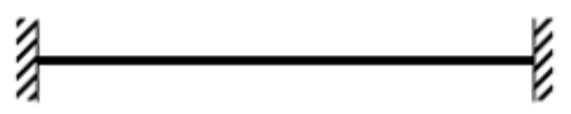

(c)

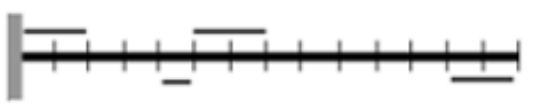

Fig. 1: Fig. 1. a) Original structure; b) Modified structure; c) modified structure

Table 1: Comparison between reference and approximate results: Eigenfrequencies relative errors in $\% ; \Delta_{M A C_{\nu}}$ values in $\%$ between the beams a) and b)

\begin{tabular}{llllll}
\hline Modes & $f_{\nu 0}^{e x}$ & $f_{\nu}^{e x}$ & $\hat{f}_{\nu}$ & $\widehat{f_{\nu}^{r e}}$ & $M A C_{\nu}$ \\
\hline 1 & 10.50 & 66.86 & 66.86 & 66.86 & 0.9999 \\
2 & 42.15 & 84.32 & 84.32 & 84.32 & 0.9999 \\
3 & 65.84 & 168.77 & 168.77 & 168.77 & 0.9999 \\
4 & 126.53 & 184.30 & 184.30 & 184.30 & 1.0000 \\
5 & 184.37 & 253.46 & 253.46 & 253.46 & 0.9999 \\
CPU & $0.0084 \mathrm{~s}$ & 0.00836 & $0.0071 \mathrm{~s}$ & $0.0065 \mathrm{~s}$ & \\
\hline
\end{tabular}

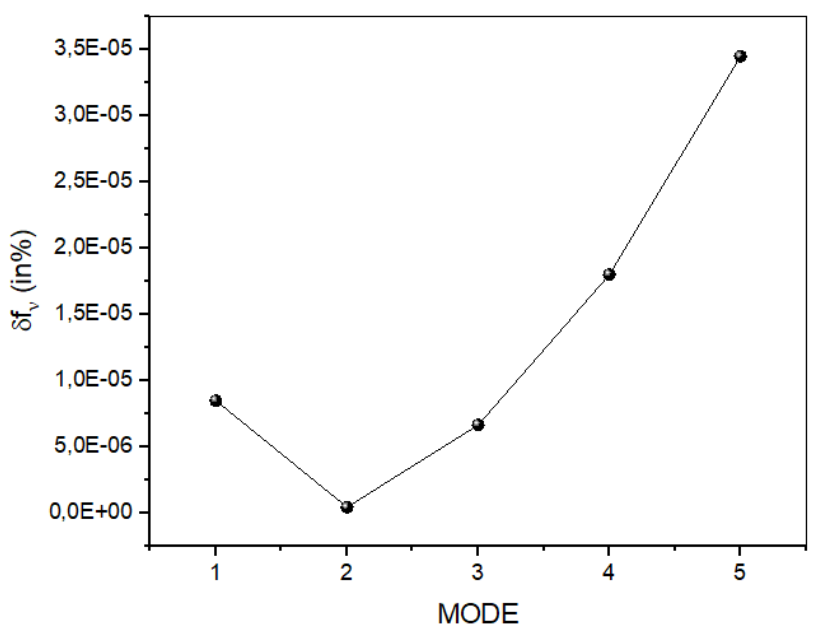

Fig. 2: Comparison between reference and approximate results: Eigenfrequencies relative errors in \%
Table 3: For a structure of 859 elements 2577 degrees of freedom

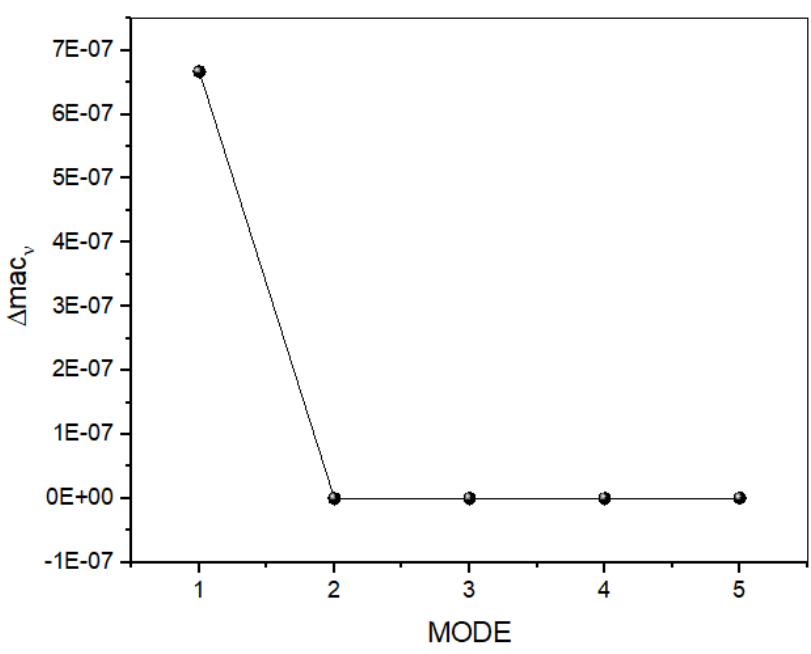

Fig. 3: Comparison between reference and approximate results: $\triangle_{M A C_{\nu}}$ values in $\%$

Table 2: For a structure of 429 elements 1287 degrees of freedom

\begin{tabular}{llllll}
\hline Modes & $f_{\nu 0}^{e x}$ & $f_{\nu}^{e x}$ & $\hat{f}_{\nu}$ & $\widehat{f_{\nu}^{r e}}$ & $M A C_{\nu}$ \\
\hline 1 & 10.50 & 65.88 & 66.83 & 66.86 & 1.0000 \\
2 & 42.15 & 84.32 & 84.30 & 84.31 & 0.9999 \\
3 & 65.84 & 168.62 & 168.62 & 168.62 & 0.9999 \\
4 & 126.53 & 184.55 & 184.00 & 184.30 & 1.0000 \\
5 & 184.37 & 252.93 & 252.93 & 252.93 & 0.9999 \\
CPU & $10.721 \mathrm{~s}$ & $10.556 \mathrm{~s}$ & $3.708 \mathrm{~s}$ & $0.986 \mathrm{~s}$ & \\
\hline
\end{tabular}

\begin{tabular}{llllll}
\hline Modes & $f_{\nu 0}^{e x}$ & $f_{\nu}^{e x}$ & $\hat{f}_{\nu}$ & $\widehat{f_{\nu}^{r e}}$ & $M A C_{\nu}$ \\
\hline 1 & 10.50 & 89.67 & 66.31 & 66.86 & 0.6898 \\
2 & 42.15 & 100.94 & 84.30 & 84.31 & 0.8899 \\
3 & 65.84 & 160.93 & 168.50 & 168.62 & 0.8599 \\
4 & 126.46 & 172.41 & 177.56 & 184.30 & 0.7999 \\
5 & 184.37 & 251.03 & 252.86 & 252.93 & 0.7639 \\
$\mathrm{CPU}$ & $88.832 \mathrm{~s}$ & $88.787 \mathrm{~s}$ & $25.877 \mathrm{~s}$ & $6.883 \mathrm{~s}$ & \\
\hline
\end{tabular}

Table 4: Comparison between reference and approximate results: Eigenfrequencies relative errors in \%; $\Delta_{M A C_{\nu}}$ values in $\%$ between the beams a) and b)

\begin{tabular}{llllll}
\hline \multirow{2}{*}{ Modes } & $f_{\nu 0}^{e x}$ & $f_{\nu}^{e x}$ & $\hat{f}_{\nu}$ & $\widehat{f_{\nu}^{r e}}$ & $M A C_{\nu}$ \\
\hline 1 & 10.50 & 3.90 & 3.90 & 3.90 & 1.000 \\
2 & 42.15 & 22.50 & 22.50 & 22.50 & 0.9999 \\
3 & 65.84 & 41.83 & 41.83 & 41.83 & 1.000 \\
4 & 126.53 & 121.65 & 121.67 & 121.67 & 0.9999 \\
5 & 184.37 & 122.87 & 122.87 & 122.87 & 1.000 \\
$\mathrm{CPU}$ & $0.0085 \mathrm{~s}$ & $0.0084 \mathrm{~s}$ & $0.0081 \mathrm{~s}$ & $0.0075 \mathrm{~s}$ & \\
\hline
\end{tabular}




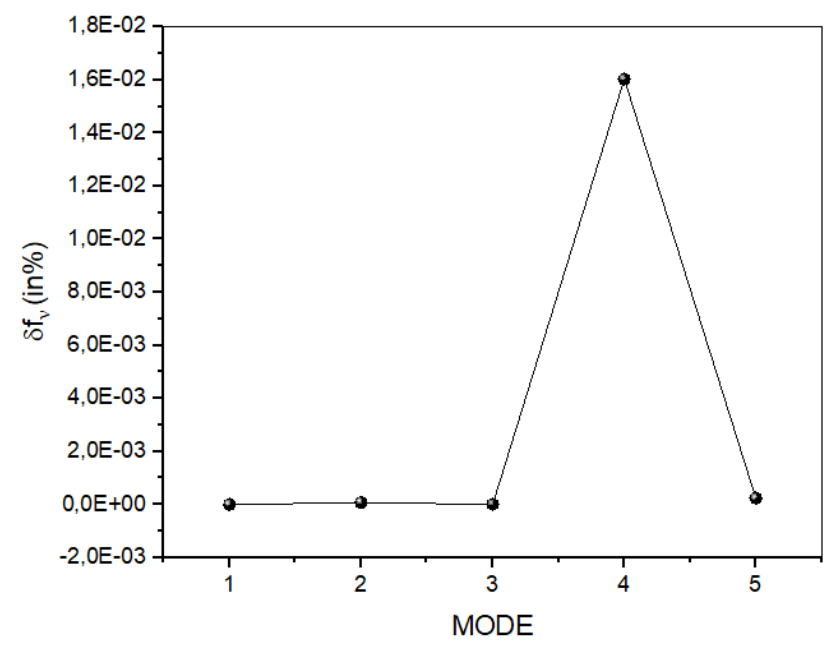

Fig. 4: Comparison between reference and approximate results: Eigenfrequencies relative errors in \%

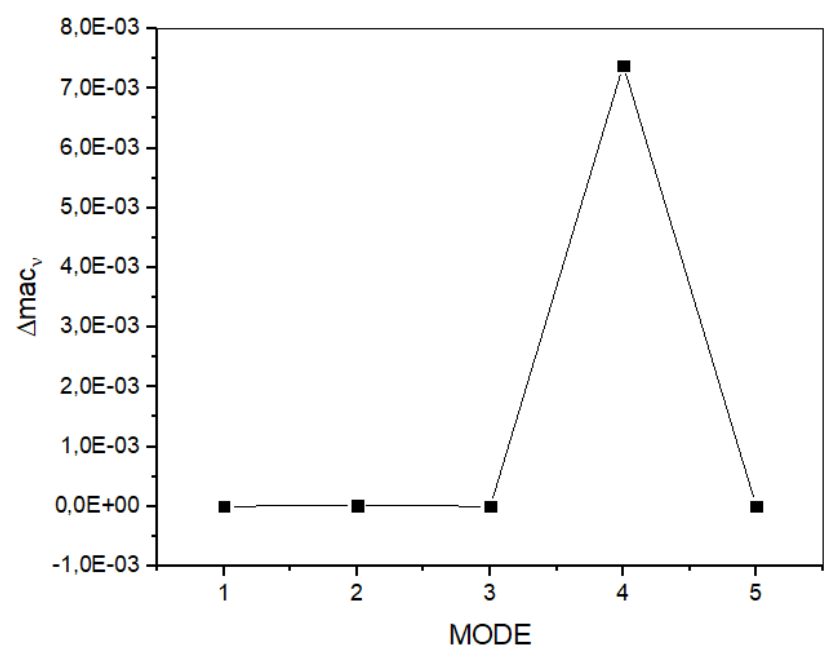

Fig. 5: Comparison between reference and approximate results: $\Delta_{M A C_{\nu}}$ values in $\%$

Table 5: The natural frequencies of the structure (c which contains 429 elements in which there are 210 elements to modify and 1287 degrees of freedom

\begin{tabular}{llllll}
\hline Modes & $f_{\nu 0}^{e x}$ & $f_{\nu}^{e x}$ & $\hat{f}_{\nu}$ & $\widehat{f}_{\nu}^{r e}$ & $M A C_{\nu}$ \\
\hline 1 & 10.50 & 3.91 & 3.91 & 3.91 & 1.000 \\
2 & 42.15 & 22.88 & 22.88 & 22.88 & 1.000 \\
3 & 65.84 & 41.85 & 41.86 & 41.86 & 0.9999 \\
4 & 126.46 & 123.08 & 123.08 & 123.08 & 1.000 \\
5 & 184.37 & 123.30 & 123.32 & 123.32 & 0.9999 \\
$\mathrm{CPU}$ & $11.564 \mathrm{~s}$ & $11.821 \mathrm{~s}$ & $4.080 \mathrm{~s}$ & $1.535 \mathrm{~s}$ & \\
\hline
\end{tabular}

Table 6: The natural frequencies of the structure (c which contains 859 elements in which there are 210 elements to modify and 2577 degrees of freedom

\begin{tabular}{llllll}
\hline Modes & $f_{\nu 0}^{e x}$ & $f_{\nu}^{e x}$ & $\hat{f}_{\nu}$ & $\widehat{f_{\nu}^{r e}}$ & $M A C_{\nu}$ \\
\hline 1 & 10.50 & 4.32 & 4.29 & 4.30 & 1.0000 \\
2 & 42.15 & 42.40 & 42.40 & 42.40 & 0.9999 \\
3 & 65.84 & 44.02 & 44.02 & 44.02 & 0.9998 \\
4 & 126.46 & 116.06 & 116.05 & 116.06 & 0.9996 \\
5 & 184.37 & 125.78 & 125.78 & 125.78 & 1.0000 \\
CPU & $88.392 \mathrm{~s}$ & $88.569 \mathrm{~s}$ & $28.105 \mathrm{~s}$ & $7.161 \mathrm{~s}$ & \\
\hline
\end{tabular}

\section{CONCLUSiON}

In this work, the obtained results starting from the problem of the reduced linear symmetric eigenvalues, are of very high quality. This proves that the proposed method uses less arithmetic operations and storage space, taking full advantage of linearity, symmetry and sparse matrices. When the database is identified, we do not need to know the matrices of the original structure, ultimately it can contribute significantly to reducing the time and cost of developing a prototype and updating models with identified data. And it can be easily implemented with any general purpose finite element analysis program.Unlike methods based on a combined block approximation with shifting, where the calculation of the upper modes is done using triangular factorizations,or others focused on the topological modifications by adding DOFs.

\section{REFERENCES}

[1] KB. Elliott AND LD. Mitchell, The effect of modal truncation on modal modification, Proceedings of the 5th International Modal Analysis Conference, London, Imperial College-SEM , (1987): p72.

[2] G. Coupry, Forecast of the consecutive variation of the modes to the modification of a structure, Aerospace Research Journal , 3(1973): p173.

[3] YM. RAM AND SG. BRAUn, An inverse problem associated with modification of incomplete dynamic systems, International Journal of Analytical and Experimental Modal Analysis , 6(1991): p201.

[4] S. Cogan, Reanalysis of the Structures Starting from Experimental Data: Rigidification and Substitution, Besançon, France, Franche-Comté University, (1990).

[5] BP. Wang AND S. CAldwell and C. Smith, Finite Elements in Analysis and Design, 12(1992): p191.

[6] H. Ait Rimouch, Analysis of Modified Mechanical Structures, Besançon, France, Franche-Comté University, (1993). Electron., submitted for publication.

[7] R. Sohaney And D. Bonnecase, Residual mobilities and structural dynamic modifications, IMAC7 Ed.: Demichele, D. J. New York, Elsevier Science and Technology Books, (1989): p568.

[8] R.N.Coppolino, Structural mode sensitivity to local modification, Journal , (1981): p3441. 
[9] P. L. Jezequel, Procedure to reduce the effects of modal truncation in eigensolutionreanalysis, AIAA Journal , 28(1990): p896-p902.

[10] I. G. Burova, N. S. Domnin, A. E. Vezhlev, A. V. Lebedeva, A. N. Pakulina, Roman Jasek, On the Solution of the Fredholm Equation of the Second Kind, WSEAS Transactions on Mathematics, pp. 319-328, Volume 17, (2018).

[11] I. G. Burova, V. M. Ryabov, M. A. KalnitSKAIA, A. V. MALEVICH, The Interpolation Method for Calculating Eigenvalues of Matrices,WSEAS Transactions on Systems and Control, pp. 104-111, Volume 14, (2019).

[12] S. P. Zheng, B. S. Wu, AND Z. G. LI Vibration reanalysis based on block combined approximations with shifting, Comput. Struct., vol. 149, pp. 72-80, Mar. (2015), doi: 10.1016/J.COMPSTRUC.2014.12.006.

[13] S. P. Zheng, B. S. Wu, And Z. G. Li Free vibration reanalysis of structures with added degrees of freedom, Comput. Struct., vol. 206, pp. 31-41, Aug. (2018), doi: 10.1016/J.COMPSTRUC.2018.06.006.

[14] Ouadie Koubaiti, Jaouad El-MekKaoui, And Ahmed Elkhalfi. Complete study for solving Navier-Lamé equation with new boundary condition using mini element method, International journal of mechanics, Volume 12, Pages: 46-58. (2018).

[15] Ouadie Koubaiti, Ahmed Elkhalfi, Jaouad El-Mekaoui, Nikos Mastorakis. Solving the problem of constraints due to Dirichlet boundary conditions in the context of the mini element method, International journal of mechanics, Volume 14, Pages: 12-20. (2020).

[16] Ouadie Koubaiti, Jaouad El-mekKaOui, And Ahmed Elkhalfi Elasticity with mixed finite element. Communications in Applied Analysis. vol: 22. No: 4. (2018).

[17] Ouadie Koubaiti, Ahmed Elkhalfi, Jaouad EL-MEKKAOUI WEB-Spline Finite Elements for the Approximation of Navier-Lam System with $C_{A, B}$ Boundary Condition Abstract and Applied Analysis, vol. 2020, Article ID 4879723, 14 pages. https://doi.org/10.1155/2020/4879723. (2020).

[18] R. B. Nelson, Simplified calculation of eigenvector derivatives, AIAA Journal, 14(1976): p1201-p1205.

[19] J. C. Chen AND B. K. WADA, Matrix perturbation for structural dynamic analysis, AIAA Journal, 15(1977): p1095-1100

[20] R. L. Fox AND M. P. Kapoor, Rates of change of eigenvalues and eigenvectors, AIAA Journal, 12(1968): p2426-2429.

[21] I. GohberG, P. Lancaster And L. RodMAN, Matrix Polynomials, Academic Press (1982).

[22] P. LAnCAster, Nonlinear in Parameter, A Review of Numerical Methods for Eigenvalue Problems (1977), Numerik und Andwendungen von Eigenwertaufgaben und Verzweigungsproblemen (edited by E. Bohl, L. Collatz and K. P. Hedeler) ISNM 38, BaselStuttgart, Birkhauser.
[23] G. Peters and J. H. Wilkinson, $A x=\lambda B x$ and the Generalized Eigenproblem, SIAM Journal of Numerical Analysis, Vol.7, p. 479-492, (1970).

[24] A. RuHE,Algorithms for the Non-Linear Eigenvalue Problem, SIAM Journal of Numerical Analysis, vol. 10, p. 674-689, (1973)

[25] H. Henderson and V. Harold and SR. SEARLE, On deriving the inverse of a sum of $m a-$ trices, Siam Review, 23(1981): p53-p60.

[26] H. Henderson, , Journal of Statistical Computation and Simulation, 12(1981): p138.

[27] AS. Householder, The theory of matrices in numerical analysis, Blaisdell Publ, Co., New York, (1964).

\section{Creative Commons Attribution License 4.0 (Attribution 4.0 International, CC BY 4.0)}

This article is published under the terms of the Creative Commons Attribution License 4.0

https://creativecommons.org/licenses/by/4.0/deed.en US 